\title{
TI.42.1
}

\section{Charter for a Strong Identity Proofing Profile Working Group}

- PDF: MIPWG-CharterforaStrongldentityProofingProfileWorkingGroup-150616-2216-4172.pdf

- Text: MIPWG-CharterforaStrongldentityProofingProfileWorkingGroup-150616-2216-4172.txt

\section{More Information}

\begin{tabular}{|l|l|}
\hline Repository ID & TI.42.1 \\
\hline Persistent URL & http://doi.org/10.26869/TI.42.1 \\
\hline Title & Charter for a Strong Identity Proofing Profile Working Group \\
\hline Authors & Multi-Factor Authentication Interoperability Profile Working Group \\
\hline Sponsor & InCommon AAC \\
\hline Review & \\
\hline Status & Legacy \\
\hline Publish Date & June, 2016 \\
\hline DOI & $10.26869 /$ TI.42.1 \\
\hline Signature & \\
\hline Deprecated & No \\
\hline Future Review & \\
\hline Supersedes & \\
\hline Format & PDF \\
\hline Related Docs & TI.36.1, TI.37.1, TI.38.1, TI.39.1, TI.40.1, TI.41.1 \\
\hline Development Location & \\
\hline IP Framework & \\
\hline Subject Tags & \\
\hline Notes & \\
\hline & \\
\hline
\end{tabular}

